\title{
Correction to: Patient-Reported Outcomes in an Observational Cohort of HIV-1-Infected Adults on Darunavir/Cobicistat-Based Regimens: Beyond Viral Suppression
}

\author{
Andrea Antinori ${ }^{1} \cdot$ Maria V. Cossu $^{2} \cdot$ Barbara Menzaghi $^{3} \cdot$ Gaetana Sterrantino $^{4} \cdot$ Nicola Squillace $^{5}$. \\ Valentina Di Cristo ${ }^{6}$. Annamaria Cattelan ${ }^{7}$. Emanuele Focà ${ }^{8}$. Antonella Castagna ${ }^{9,10}$. Giancarlo Orofino ${ }^{11}$. \\ Daniela Valenti ${ }^{12}$. Gabriella D'Ettore ${ }^{13}$. Lucia Aprea ${ }^{14}$. Sergio Ferrara ${ }^{15}$. Maria E. Locatelli ${ }^{16}$. Giordano Madeddu ${ }^{17}$. \\ Emanuele Pontali ${ }^{18} \cdot$ Paolo Scerbo $^{19} \cdot$ Barbara Rossetti $^{20}$ - Alessia Uglietti $^{21} \cdot$ Roberta Termini $^{21}$. Francesco Rucci ${ }^{22}$. \\ Andrea Gori ${ }^{23,24}$. Daniela Mancusi ${ }^{21}$
}

Published online: 29 May 2020

(c) The Author(s) 2020

\section{Correction to: \\ The Patient-Patient-Centered Outcomes Research \\ (2020) 13:375-387 \\ https://doi.org/10.1007/s40271-020-00413-y}

In the original version of this article, in Fig. $2 b$ the formatting on the $\mathrm{x}$-axis of the graph has been published incorrectly.

The original article can be found online at https://doi.org/10.1007/ s40271-020-00413-y.

Daniela Mancusi

dmancusi@its.jnj.com

1 HIV/AIDS Department, National Institute for Infectious Diseases "Lazzaro Spallanzani"-IRCCS, Rome, Italy

2 1st Division of Infectious Diseases, ASST Fatebenefratelli Sacco, Milan, Italy

3 Infectious Diseases, Azienda Socio-Sanitaria Territoriale della Valle Olona-Busto Arsizio, Busto Arsizio, Italy

4 Division of Tropical and Infectious Disease, Department of Clinical and Experimental Medicine, University of Florence, Florence, Italy

5 Clinic of Infectious Diseases, "San Gerardo" Hospital, ASST Monza, Monza, Italy

6 DIBIC Luigi Sacco, Division of Infectious Diseases, University of Milan, Milan, Italy

7 Division of Infectious and Tropical Diseases, Azienda Ospedaliero-Universitaria di Padova, Padua, Italy

8 Department of Infectious and Tropical Diseases, University of Brescia and Spedali Civili General Hospital, Brescia, Italy

9 Department of Infectious Diseases, IRCSS San Raffaele Scientific Institute, Milan, Italy

10 Università Vita-Salute San Raffaele, Milan, Italy

11 Unit of Infectious Diseases, Amedeo di Savoia Hospital, Turin, Italy
12 FROM Research Foundation, Papa Giovanni XXIII Hospital, Bergamo, Italy

13 Department of Public Health and Infectious Disease, Sapienza University, Rome, Italy

14 VIII Divisione di Malattie Infettive, A.O.R.N. Cotugno, Naples, Italy

15 Department of Clinical and Experimental Medicine, Infectious Diseases Unit, University of Foggia, Foggia, Italy

16 UOC Malattie Infettive, ARNAS “Garibaldi”, Catania, Italy

17 Unit of Infectious Diseases, Department of Medical, Surgical and Experimental Sciences, University of Sassari, Sassari, Italy

18 Infectious Diseases, E.O. Ospedali Galliera, Genoa, Italy

19 Unit of Infectious Diseases, "Pugliese-Ciaccio" Hospital, Catanzaro, Italy

20 Infectious Diseases Unit, Siena University Hospital, Siena, Italy

21 Medical Affairs Department, Infectious Diseases, Janssen-Cilag SpA, Via Michelangelo Buonarroti, 23, Cologno Monzese, 20093 Milan, Italy

22 Department of Oncology and Onco-Hematology, Postgraduate School of Clinical Pharmacology and Toxicology, University of Milan, Milan, Italy

23 Infectious Diseases Unit, Fondazione IRCCS Ca' Granda, Ospedale Maggiore Policlinico, University of Milan, Milan, Italy

24 Department of Infectious Diseases, San Gerardo Hospital, University of Milano-Bicocca, Monza, Italy 
Previously published as

b

Selected Items (HIV-TSQc) - Visit 2 and Visit 4

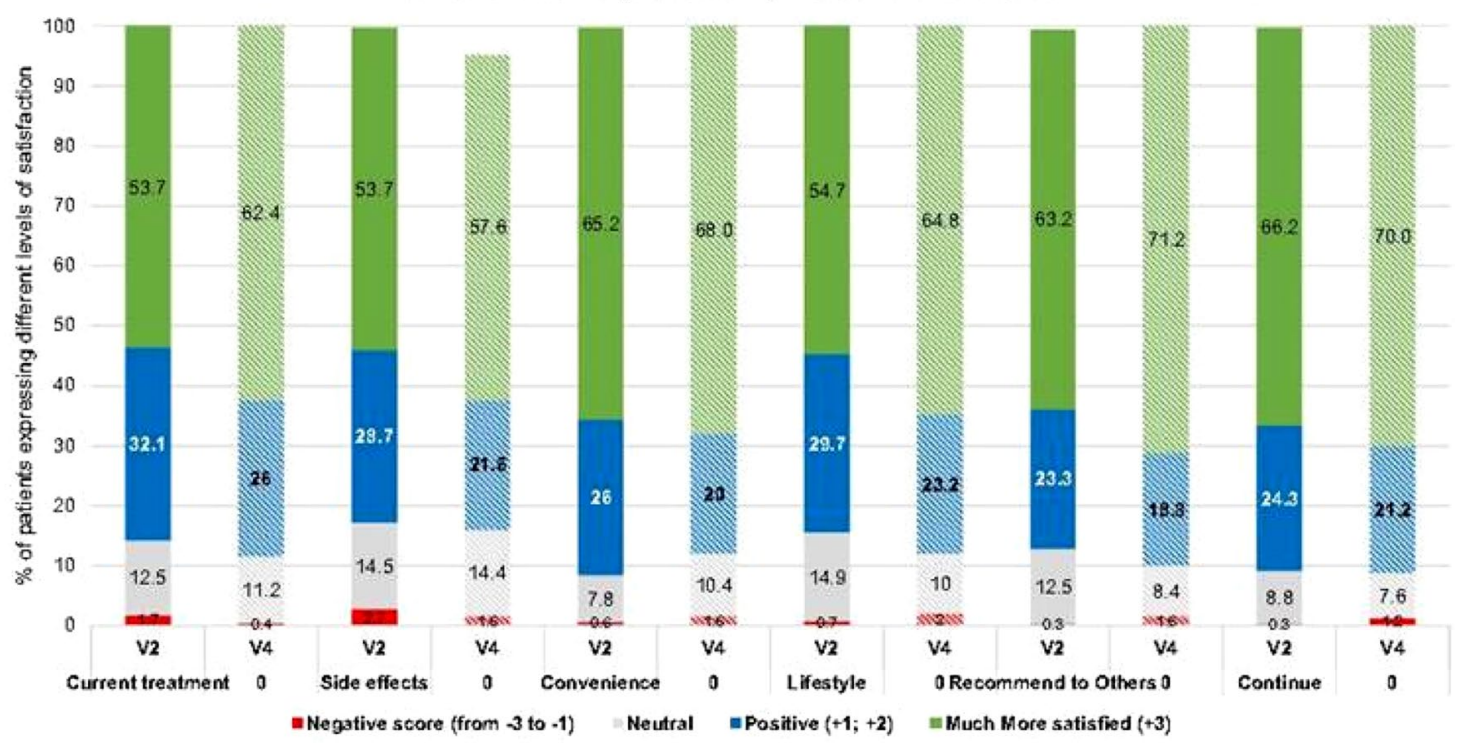

It should be published as

Selected Items (HIV-TSQC) - Visit 2 and Visit 4

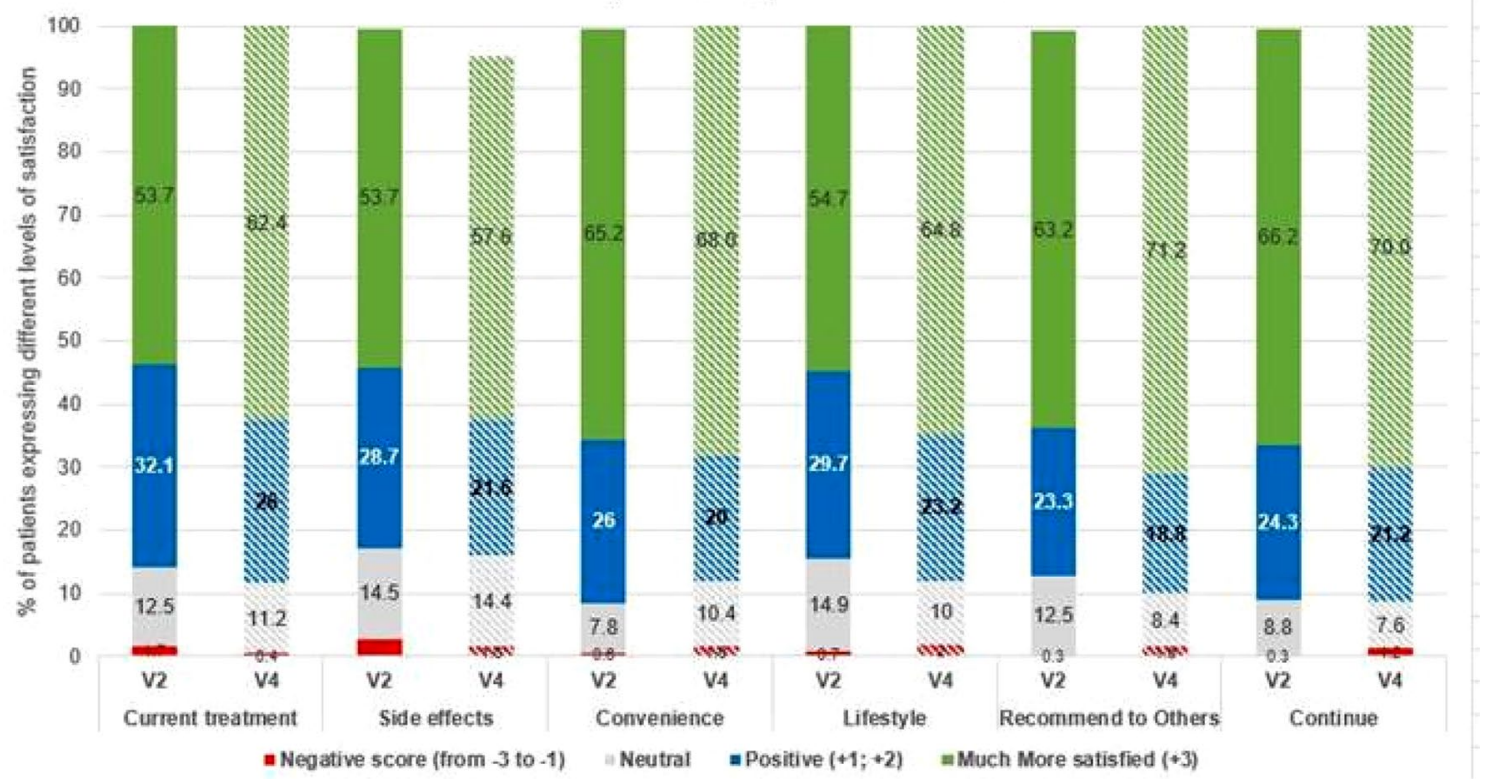

Open Access This article is licensed under a Creative Commons Attribution-NonCommercial 4.0 International License, which permits any noncommercial use, sharing, adaptation, distribution and reproduction in any medium or format, as long as you give appropriate credit to the original author(s) and the source, provide a link to the Creative Commons licence, and indicate if changes were made. The images or other third party material in this article are included in the article's Creative Commons licence, unless indicated otherwise in a credit line to the material. If material is not included in the article's Creative Commons licence and your intended use is not permitted by statutory regulation or exceeds the permitted use, you will need to obtain permission directly from the copyright holder. To view a copy of this licence, visit http://creativecommons.org/licenses/by-nc/4.0/. 